\title{
Carnets
}

Revue électronique d'études françaises de l'APEF

Deuxième série - 6 | 2016

Exotopies de Barthes

\section{Barthes au Brésil : auteur « difficile » et libertaire}

\section{Laura Brandini}

\section{(2) OpenEdition}

\section{Journals}

Édition électronique

URL : http://journals.openedition.org/carnets/730

DOI : $10.4000 /$ carnets. 730

ISSN : 1646-7698

Éditeur

APEF

\section{Référence électronique}

Laura Brandini, «Barthes au Brésil : auteur « difficile » et libertaire », Carnets [En ligne], Deuxième série 6 | 2016, mis en ligne le 31 janvier 2016, consulté le 02 mai 2019. URL : http:// journals.openedition.org/carnets/730 ; DOI : 10.4000/carnets.730

Ce document a été généré automatiquement le 2 mai 2019.

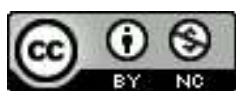

Carnets est mis à disposition selon les termes de la licence Creative Commons - Atribution - Pas d'utilisation commerciale 4.0 International. 


\section{Barthes au Brésil : auteur « difficile » et libertaire}

\section{Laura Brandini}

1 Dans une année où l'on fête partout le centenaire de naissance de Roland Barthes, rien de plus approprié qu'un colloque sur « Barthes intériorisé » pour s'interroger sur les usages les plus différents dont son œuvre a fait objet, dans un passé récent, et fait objet, au présent. Antoine Compagnon, dans son article «Lequel est le bon?», s'occupe de la pluralité des Barthes dont l'œuvre barthésienne est faite, chaque nouveau projet d'écriture conduisant l'écrivain dans une direction distincte de celle prise auparavant, ce qui naturellement déroutait ses disciples. La question de Compagnon - qui est celle des admirateurs de Barthes - peut être adaptée au but de mon article : Barthes, lequel est le bon, au Brésil ? Autrement dit, quel Barthes a été intériorisé dans ce pays où il n'a jamais été?

2 La réponse peut être retrouvée dans les pages des journaux : pour occasion du centenaire de parution du Degré zéro de l'écriture, une grande exposition consacrée à Barthes a eu lieu au Centre Georges Pompidou, à Paris, entre 2002 et 2003. L'évènement a entraîné la publication de plusieurs articles dans le journal brésilien O Estado de S. Paulo, le plus grand $\mathrm{du}$ pays en numéro d'exemplaires et en temps de circulation. Gilles Lapouge, collaborateur hebdomadaire du journal depuis 1951, a écrit, à l'époque, à propos de Barthes revisité par l'exposition:

À mon avis, cette exposition dissipe un formidable malentendu qui a été créé autour de Barthes pendant son existence : très tôt flatté et admiré, professeur charmant, d'une belle et douce voix, il a été "congelé» dans le rôle de "théoricien ", de "sémiologue ». On a fait de lui - lui-même l'a fait de soi - un théoricien rigoureux, austère, un homme de la «structure » ou du «syntagme ", le grand amateur des néologismes ou des citations grecques et du jargon; en somme, un pédant séducteur.

Des générations d'étudiants se sont évanouies en face de ses études stylistiques ou structurelles. Aujourd'hui, lorsqu'on relit ces études, ces lourdes « leçons doublées de châtiment » nous tombent des mains : une étude comme Le Système de la mode est lancinante, dépourvue de tout intérêt, aussi bien que mal conduite dans le plan de la théorie. 
Mais le vrai Barthes était bien différent. (...)

Très heureusement, c'est l'autre Barthes - un Barthes libre, joyeux, plaisant et insolent, jovial et intrépide, passionnément libre de toute théorie, amateur du monde et des gens - que l'exposition a décidé d'exalter, non pas le Barthes enseveli par Marx et, surtout, par Saussure, ou par la théorie structuraliste. (2003 : D1, je traduis)

Suite à la visite de l'exposition, Lapouge distingue deux Barthes : celui du structuralisme qui terrorisait les étudiants, et l'écrivain «libre de toute théorie »; donc deux auteurs posés dans des pôles contraires, l'un étant le faux, l'autre, "le vrai», l'un, l'auteur « difficile », l'autre, le « libertaire».

4 Pour plus superficielle et forcée qu'elle puisse l'être, cette dichotomie résume bien les points de vue sur Barthes, au Brésil, aussi bien que dans d'autres pays ${ }^{1}$ : un auteur de lecture difficile - surtout à cause des œuvres issues d'un structuralisme dur, comme Éléments de sémiologie (1965), «Introduction à l'analyse structurale des récits » (1966), Le Système de la mode (1967) -, aussi bien qu'un écrivain inspiré et inspirateur - je pense notamment au Roland Barthes par Roland Barthes (1975), à Fragments d'un discours amoureux (1977) et à Leçon (1978).

5 Or, plus de vingt ans auparavant, la perception de l'œuvre de Barthes n'était pas la même : le jeudi 27 mars 1980, le journal affiche à la Une sa disparition. Une page regroupe sous le titre "Os Símbolos perdem Roland Barthes» [Les Symboles perdent Roland Barthes], une brève présentation de Barthes et un reportage sur sa mort. Trois professeurs universitaires brésiliens témoignent de l'importance de son œuvre. Ce qui en ressort est l'image du grand sémiologue structuraliste; une œuvre est citée deux fois : Éléments de sémiologie.

6 À ce moment-là, le Barthes qui circulait au Brésil était toujours l'auteur structuraliste, grâce aux traductions de ses œuvres des années 50 et 60, parues depuis 1970. Dans cette décennie sont traduits : un recueil des Essais critiques publié avec Critique et vérité (1970), traduit par initiative de l'introductrice de Barthes au Brésil, Leyla Perrone-Moisés, Le Degré zéro de l'écriture, Éléments de sémiologie et «Introduction à l'analyse structurale des récits » (1971), Mythologies (1972), Le Plaisir du texte et Roland Barthes par Roland Barthes (1977) et Le Système de la mode (1979). Exceptés Le Plaisir du texte et Roland Barthes par Roland Barthes, tous ces livres témoignent de l'enthousiasme de Barthes pour la linguistique, soit dans sa forme saussurienne (Le Degré zéro, Mythologies), sémiologique ( Éléments de sémiologie) ou tout simplement structuraliste («Introduction à l'analyse structurale des récits », Le Système de la mode). En consonance avec les débats français et, surtout, à cause des choix de traduction - qui, à leur tour, suivent les exigences du système culturel cible -, l'écrivain, dans les années 70, est inséparable du structuralisme et, d'après son mot, il est « l'imago de la sémiologie » (Barthes, 2002, v. $4: 522$ ).

7 Cette imago qui a représenté Barthes tant en France qu'au Brésil, a été le sujet de nombreuses polémiques et la cible des attaques des intellectuels contraires au structuralisme, dont le plus éminent est José Guilherme Merquior. Celui-ci présente dans son essai «O Estruturalismo dos pobres » [ « Le structuralisme des pauvres»] les critiques les plus importantes que cette " activité » a reçu de la part des intellectuels brésiliens. Le paragraphe d'introduction de son essai donne le ton:

Si vous voulez poursuivre des études universitaires en lettres, préparez-vous: quelle idée vous vous faites, pas encore du métalangage, mais du moins de la grammaire générative du code poétique ? Quelle est votre opinion sur les résultats, dans la tâche de mettre en équation la littérarité du poématique, des microscopies 
montées sur la formule poésie de la grammaire/ grammaire de la poésie ? Combien d'actants vous êtes capable de distinguer dans la textualité des romans que vous avez probablement (re-)lu ? Et qu'est-ce que vous me dites du « pluriel du texte » de Barthes - est-il possible de l'assimiler au génotexte de la fameuse Kristeva ? Vous vous considérez en condition de discerner le travail signifiant dans le nouveau roman, par exemple, par le moyen d'une "décodification " "sémanalytique » de bases glossématiques? Ou bien préférez-vous poursuivre la "signifiance", assujettie à quelques coupures épistémologiques, dans le terrain de la forclusion, si clairement exposée dans l'archipédant séminaire de Lacan? (Merquior, 1975 : 07)

L'auteur établit d'emblée un dialogue avec un futur étudiant hypothétique tout en versant sur lui un torrent de termes, expressions et concepts créés ou divulgués à l'époque par les courants structuralistes. Presque tout y est, ni Barthes n'y échappe, avec une allusion à $S / Z$, où il défend sa compréhension du texte littéraire en tant qu'une "galaxie de signifiants» (2002, v. $3: 123)$. Face à la complexité des questions posées, l'étudiant, étourdi, ne peut qu'abandonner ses aspirations aux études des lettres...

L'effet comique, obtenu par l'entassement de vocabulaire technique, a pour but d'attaquer le structuralisme dans ce qu'il prise le plus dans ses objets : le langage. L'essai poursuit les attaques par la critique au langage structuraliste dans tous ses aspects, à savoir, la variété des courants théoriques, chacune imposant son vocabulaire particulier le courant ethnographique de Lévi-Strauss, marxiste d'Althusser ou psychanalytique de Lacan, par exemple -, la pluralité des conceptions du terme structure, les traductions du concept d'écriture - en portugais du Brésil, « escrita » ou « escritura»? -, concluant ses arguments avec ce qu'il considère la caractéristique principale du structuralisme: le «terrorisme terminologique » $(1975: 08)$

10 Barthes, grand représentant du structuralisme, n’a pas échappé aux attaques au langage, très communs dans la presse brésilienne, adressés plutôt à une science alors nouvelle, peu connue et incomprise. À cet égard, l'article de Maria Thereza Fraga Rocco, «Linguística : ciência e deslumbramento » [Linguistique: science et éblouissement], paru dans le journal O Estado de S. Paulo en 1977, offre une réflexion importante sur le panorama des études linguistiques au Brésil. Son texte s'occupe, d'une part, des projets touchant l'enseignement de la langue portugaise dans les écoles, et d'autre part, de manière plus prononcée, des problèmes créés par le rôle de plus en plus important de la linguistique face les autres sciences humaines. Dans les mots de l'auteur,

Je crois que l'un des problèmes essentiels [de la linguistique brésilienne], c'est-àdire celui qui se réfère à un excès de complexité, a pour origine l'éblouissement causé par une science nouvelle ayant la prétention d'être en avance par rapport aux autres et qui finit par devenir objet de foi, même pour ceux qui ne connaissent guère le credo. (Rocco, $1977: 4$, je traduis)

11 Par "excès de complexité " Rocco comprend le jargon développé par les théories linguistiques afin de défendre leurs arguments et de formuler leurs concepts. L'« éblouissement » auquel l'auteur fait référence est l'effet provoqué par l'ascension de la science linguistique au niveau d'une religion, dans une union pour le moins inattendue. La linguistique, science de base du structuralisme, était elle aussi accusée de «terrorisme terminologique », mais dans des termes plus subtils :

Trop préoccupés de l'emploi d'une nomenclature inaccessible et avec la prétention de voir les faits transformés en science rien qu'avec ce geste, ces groupes [d'intellectuels structuralistes] finissent par divorcer entièrement du réel, $d u$ possible, lorsqu'ils deviennent des blocs froids, hermétiques, identifiables uniquement par la terminologie complexe, indéchiffrable et parfois inappropriée 
(même si les phénomènes auxquels ils font appel traitent du même homme qui pense et parle de façon organisée) (ibid. : 4)

L'auteur suggère que la linguistique, élevée à la condition d'une science à la mode, provoque un « éblouissement » capable d'aveugler beaucoup d'intellectuels. Ceux-ci, dans l'élan de mettre en pratique de nouvelles théories, avec le but de montrer qu'ils sont à la page et bien informés dans leurs domaines d'études, appliquent souvent le jargon linguistique et ses modèles analytiques de manière tout à fait aliénée par rapport aux particularités de leurs objets. De ce processus vite conduit, dont les résultats intéressent moins que la démonstration technique, Rocco souligne son côté le plus visible : le langage codé qui permet seulement à de très peu élus l'accès aux nouvelles théories linguistiques.

Une telle perception était récurrente en France aussi, Barthes en étant également la cible, par exemple, en 1978, dans le Roland Barthes sans peine, de Michel-Antoine Burnier et Patrick Rambaud. Dans ce livre les auteurs font un pastiche du langage de l'écrivain et le nomment « Roland-Barthes » :

Le R.B. (en Roland-Barthes, Roland Barthes se dit R.B.) apparut sous sa forme archaïque voilà vingt-cinq ans, dans l'ouvrage intitulé Le degré zéro de l'écriture. Depuis, il s'est peu à peu détaché du français dont il est partiellement issu, se constituant comme langage autonome avec sa grammaire et son vocabulaire propres. (Burnier, Rambaud, $1978: 07$ )

14 Face une langue étrangère, les auteurs offrent des exemples de phrases employées dans des situations de dialogue, tout comme dans un guide pratique de conversation:

1 - "Salut! Salut !" Tel est, irrépressible, rabâché, le salve qui suture notre distance logosphérique.

Français : Bonjour, cher ami, comment ça va?

2 - “J'intériorise votre apostrophage (le mangeur d'apostrophes ?) en (l'in)versant dans le positif.

Français : Ça va bien, merci.

3 - Le sereno coelo, c'est la réification actuelle d'un il-fait-drôlement-bon, "comme si de rien n'était".

Français : Quel beau temps!

4 - La libido phoebale se théorise/ rentabilise irréductiblement sous forme jubilatoire.

Français : Oui, le soleil est vraiment splendide. (Burnier, Rambaud, 1978 : 19, les auteurs soulignent)

15 Le contraste entre l'obscurité du « Roland-Barthes » et la frugalité de la langue française fait rire. Cependant, pratiquer une langue propre n'est pas le privilège de Barthes, selon les auteurs qui, dans l'introduction du livre, s'excusent de ne pas s'occuper des autres langues structuralistes :

P.S. Nous ne traiterons pas des idiomes proches du R.B., le Jacques-Derrida, le Philippe-Sollers, le Lacan, plus sévères et moins riches. Nous négligerons également les patois comme le Guattaro-Deleuzisme, le Ricardou et le Cixous. Enfin nous n'entrerons pas dans la polémique sur les influences réciproques de ces divers idiomes. (Burnier, Rambaud $1978: 13$, les auteurs soulignent)

Même si ce livre n'ait pas été noté dans la presse brésilienne, les critiques au langage structuraliste faites en France arrivaient au Brésil. Et les œuvres de Barthes, dans les années 70, alors connues, étaient discutées - quand elles n'étaient pas carrément démolies - en tant que des écrits appartenant à une école critique : moins par leurs qualités et défauts, ou par les particularités de son structuralisme, classé comme «ondoyant et subtile" par François Dosse (Dosse, 2007, v.1: 117), que par ses points communs avec un certain structuralisme, de langage inaccessible. Quoique cité 
indirectement, comme par Merquior et beaucoup d'autres, l'écrivain a survécu à la vague d'attaques des années 70 et est arrivé aux décennies suivantes, lorsqu'une nouvelle image s'est imposée.

La post-modernité instituée par les années 80-90 a connu un tout autre Barthes. Une fois guérie la « fièvre structuraliste » et grâce aux traductions brésiliennes des livres comme Leçon (1980), Fragments d'un discours amoureux (1981), La Chambre claire (1984) et les œuvres parues au Brésil à la fin des années 70, Le Plaisir du texte et Roland Barthes par Roland Barthes , les animosités contre l'écrivain ont cessé ; surtout, après sa mort, en 1980, Barthes a été canonisé. Sa conférence au Collège de France s'est transformée en libelle de lutte contre tous les types d'imposition et les autres livres des années 70 ont été lus en tant que des cris de liberté justement contre l'orthodoxie structuraliste, des autorisations à l'abandon des manuels d'analyse littéraire au profit de l'expansion du désir hédoniste du lecteur.

Barthes, donc, n'était plus critiqué, car ceux qui écrivaient dans les journaux, depuis les années 80 , les journalistes culturels et non plus les critiques, puisaient leur formation aux cours universitaires de journalisme qui avaient, à la base, la lecture de ses textes, présents dans les bibliographies universitaires depuis la décennie précédente. L'écrivain, donc, acquiert une image de libérateur de la pensée contre la prison structuraliste et en tant que tel il est reconnu par la post-modernité, qui voit dans la pluralité des sens du Texte, écrit avec un « $t$ » majuscule, déclarée en 1968 avec «La Mort de l'auteur », la porte d'entrée pour le questionnement de toute sorte d'autoritarisme - discursif et social, avec l'institution des Cultural Studies.

19 Dans la presse, depuis lors, Barthes est souvent cité par la critique culturelle comme une référence, quand il s'agit d'ajouter au texte une certaine valeur de contemporanéité et de culture. Un exemple parlant de cette fonction référentielle est l'usage que fait de Barthes le critique de musique érudite du journal 0 Estado de S. Paulo, João Marcos Coelho. Dans un article sur l'exécution de la Quatrième Symphonie de Mahler par le maestro Justin Brown, lors d'un concert de l'Orchestre Symphonique de São Paulo, le critique cite l'écrivain au premier paragraphe :

Aucune musique sur la Terre ne peut être comparée à la nôtre, chante le soprano lors du dernier mouvement de la Quatrième Symphonie de Mahler. En fait, tout comme les cantates de Bach et les symphonies de Beethoven, à partir de l'Eroica les vastes fresques symphoniques de Mahler invitent non seulement à la participation à un rituel virtuellement communautaire, aussi bien qu'elles nous avertissent, comme a dit Roland Barthes : «Écoutez, je vais jouer quelque chose que vous n'avez jamais entendu auparavant » (Coelho, 2010:D5)

Le texte suit sans aucune autre mention à Barthes ou au contexte dans lequel il dit la phrase citée. Banale, dépourvue de concept ou d'une réflexion élaborée, «Écoutez, je vais jouer quelque chose que vous n'avez jamais entendu auparavant » aurait pu être dite pour n'importe qui, capable de jouer de la musique. Mais l'auteur prétend qu'il s'agisse d'une phrase de Barthes. Comme il se passe dans la majorité des critiques journalistiques depuis les années 1990, le fait d'insérer le nom de l'écrivain dans le texte semble être plus important que de citer son œuvre. Ou, pire encore, ce geste a plus de valeur que ce qui est cité. La fonction de la citation est alors inversée et devient un prétexte pour la présence du nom : Barthes.

21 Après une longue période marquée par l'incompréhension, au début, dans les années 50 et 60 , lors de la toute première réception; cessées les attaques au structuralisme, dans les années 80 , une nouvelle ère a commencé, et qui dure jusqu'au présent, où Barthes est une 
référence, un argument d'autorité. En tant que tel, l'écrivain est devenu un auteur qu'on ne discute plus dans la grande presse, c'est-à-dire un écrivain cristallisé, certes, dans une image positive, mais qui l'immobilise, tout au contraire de ce qu'il a toujours désiré. À l'heure de son centenaire de naissance, la panoplie d'évènements qui fêtent son œuvre se montre l'occasion de faire renaître les discussions autour de l'œuvre de Barthes, afin d'essayer de répondre à la question : Barthes est-il toujours un « auteur difficile » ou bien l'image de l'écrivain libertaire occupe toute la scène ? Il y aurait-elle une troisième image à l'horizon? Laquelle est la bonne?

Peut-être la réponse est sous la plume d'Antoine Compagnon, qui écrit, dans son article déjà cité: «Il n'y a pas de premier ni de second Barthes, celui du système et celui du plaisir, parce que Sur Racine, par exemple, et la sémiologie n'incarnent pas du tout le même système: il y a autant de Barthes que de projets dans lesquels il s'est investi, chacun menant au suivant. » (Compagnon, $2002: 17)$ En somme, il y a autant de Barthes que des « intériorisations de Barthes ».

\section{BIBLIOGRAPHIE}

BARTHES, Roland (2002). Oeuvres complètes. Édition d'Éric Marty. Paris : Seuil, 5v. BRANDINI, Laura (2013). Imagens de Roland Barthes no Brasil. São Paulo/ Genebra : Universidade de São Paulo/ Universidade de Genebra (tese de doutorado).

BURNIER, Michel Antoine \& RAMBAUD, Patrick (1978). Roland Barthes sans peine. Paris : Balland. Coelho, João Marcos (22/03/2010). « A Paixão encantatória de Mahler », in O Estado de S. Paulo, Caderno 2, « Música. Erudito », p. D5.

COMPAGNON, Antoine (2002). « Lequel est le bon? ", in Marielle Macé, Alexandre Gefen (org.). Barthes, au lieu du roman. Paris : Éditions Desjonquières/ Éditions Nota Bene.

CUSSET, François (2005). French Theory. Foucault, Derrida, Deleuze et Cie et les mutations de la vie intellectuelle aux Etats-Unis. Paris : La Découverte/ Poche.

DOSSE, François (2007). História do estruturalismo. Traduction de Álvaro Cabral, révision de Márcia Mansor D’Alessio. Bauru (SP) : Edusc, 2v.

LAPOUGE, Gilles (02/02/2003). «O Melhor Barthes ficou fora da sala de aula », in O Estado de S. Paulo , Caderno 2/ Cultura, p. D1.

MACHADO, Álvaro Manuel \& PAGEAUX, Daniel-Henri (1988). Da Literatura comparada à teoria da literatura. Lisboa : Edições 70.

MERQUIOR, José Guilherme (1975). O Estruturalismo dos pobres e outras questões. Rio de Janeiro : Tempo Brasileiro.

MILLIET, Sérgio (17/11/1953). « O Grau zero da escrita », in O Estado de S. Paulo, Primeiro Caderno, p. 08.

MILLIET, Diário crítico (1981-1982). São Paulo : Martins/ Editora da Universidade de São Paulo, 10v. 
PERRONE-MOISÉs, Leyla (2005). Texto, crítica, escritura. São Paulo : Martins Fontes.

Rocco, Maria Thereza Fraga (30/01/1977). «Linguística : ciência e deslumbramento », in O Estado de S. Paulo, Suplemento Cultural, p. 4.

\section{NOTES}

1. Toujours dans "Lequel est le bon?", Antoine Compagnon souligne, à propos de cette division de l'œuvre barthésienne en deux moments: "Tel est plus ou moins le schéma que les commentateurs américains reprirent pour distinguer, chez Barthes ou chez Foucault, une rupture - dont personne n'avait jamais entendu parler en France - entre le structuralisme et le post-structuralisme» (2002: 17). Le point de vue des critiques étasuniens se fonde sur des caractéristiques acquises par les œuvres des auteurs jadis «structuralistes » afin de nommer tout un nouveau tournant de la pensée théorique française, la French Theory décrite par François Cusset (2005), moins préoccupée avec une analyse orthodoxe de ses objets, qu'intéressée à faire les Textes signifier autrement - personnellement, lira-t-on surtout. Cette distinction entre structuralisme et post-structuralisme, ou post-modernité, a été adoptée au Brésil, comme les articles sur Barthes en font preuve.

\section{RÉSUMÉS}

Pour Roland Barthes, les pensées théorique et critique et l'écriture littéraire n'ont jamais été dissociées ; sa pratique fait preuve de cette alliance et la réception aux œuvres barthésiennes au Brésil a bien accusé ce caractère double : Barthes y est considéré à la fois un auteur « difficile » et une inspiration pour les jeunes et les moins jeunes. D'une part, le souvenir des années structuralistes est bien présent; néanmoins, aujourd'hui les minuties des formules structuralistes semblent « difficiles » à ceux qui s'y initient. D'autre part, la grande presse et les essais académiques rendent hommage à l'auteur des livres plus personnels, dès Le Plaisir du texte, soit par la transformation de Barthes en argument d'autorité, soit par la production d'essais fortement inspirés de l'écriture, ce qui met en relief son côté subversif et libre des doxas. Barthes, l'auteur « difficile », et Barthes, l'écrivain libertaire, coexistent au Brésil et constituent le sujet de cet article.

For Roland Barthes, the theoretical and critical thinking and literary writing were never dissociated; his practice is evidence of that alliance and reception to Barthesians works in Brazil has accused this double character: Barthes is considered both a «difficult» writer and an inspiration for young and old. On one hand, the memory of structuralist years is very present; however, today the details of the structuralist formulas seem " difficult " to those who initiate in it. On the other hand, the popular press and academic essays pay tribute to the author of the personal books, since The Pleasure of the Text, either by transforming Barthes in an argument from authority, or by the production of essays highly inspired by writing, which highlights its subversive and free side. Barthes, the "difficult " author, and Barthes, the libertarian writer, coexist in Brazil and are the subject of this article. 
INDEX

Mots-clés : Barthes (Roland), études de réception, relations Brésil-France, critique, littérature comparée

Keywords : Barthes (Roland), reception studies, Brazil-France relations, criticism, comparative literature

\section{AUTEUR}

\section{LAURA BRANDINI}

Universidade de Londrina

laura@uel.br 